\title{
ON THE COHOMOLOGY OF STABLE TWO STAGE POSTNIKOV SYSTEMS
}

\author{
BY JOHN R. HARPER
}

Communicated by Norman Steenrod, February 2, 1970

Introduction. Let $\xi=(E, p, B, F)$ denote a two stage Postnikov system with stable $k$-invariant. We announce results about $H^{*}(\Omega E)$ as a Hopf algebra over the Steenrod algebra. Mod 2 cohomology is used exclusively. Unexplained notation is from [4] and [5]. I am grateful to D. Anderson, W. Massey, F. Peterson and H. Salomonsen for many useful remarks.

We make the following assumptions on $\xi$, in addition to those of [5, p. 38]. $F$ and $B$ are simply connected products of finitely many Eilenberg-MacLane spaces. The nonzero homotopy groups of the factors of $B$ are infinite cyclic or cyclic of order $2^{k}, k=1,2, \ldots$. All factors of $F$ have $Z_{2}$ (cyclic group of order 2) as their only nonzero homotopy group.

Results of [3], [4], [5] and [8] give $H^{*}(\Omega E) \cong R \otimes U\left(X^{\prime}\right)$. The isomorphism is as algebras over $Z_{2}$ and $\otimes$ is over $Z_{2} . R=R(\Omega \xi)$ $=H^{*}(\Omega B) / \operatorname{ker} \Omega p^{*}$ and $X^{\prime}=X^{\prime}(\Omega \xi)$ is considered as known, [5, p. 54]. In general $H^{*}(\Omega E)$ does not split this way as a Hopf algebra over $Z_{2}$. The new result is Theorem A. It gives $H^{*}(\Omega E)$ as a coalgebra over $R$. It also gives information on the extension problem represented by the fundamental sequence of $\Omega \xi[5$, p. 54]. This use of the Hopf algebra structure is well known, [1], [5] and [6].

1. The main theorem. Consider the following diagram of unstable $A$-modules and $A$-maps. The squares are commutative.

$$
\begin{gathered}
X^{\prime}(\Omega \xi) \stackrel{\alpha}{\leftarrow} Y^{\prime \prime} / \lambda Y^{\prime \prime} \stackrel{\pi}{\leftarrow} Y^{\prime \prime} \stackrel{c}{\rightarrow} \Omega Y, \\
Y \stackrel{f^{*}}{\longrightarrow} Z \stackrel{\rho}{\longrightarrow} Z^{\prime} \\
\sigma_{B_{0}} \downarrow \quad \sigma_{B} \downarrow \quad \sigma^{\prime} \downarrow . \\
\Omega Y \underset{\Omega f^{*}}{\longrightarrow} \Omega Z \underset{\rho^{\prime}}{\longrightarrow} \Omega Z^{\prime}
\end{gathered}
$$

Here $\alpha$ is an $A$-isomorphism of degree $-1 ; \pi, \rho$ and $\rho^{\prime}$ are natural projections; $c$ is inclusion, and $\sigma^{\prime}$ is the obvious map. The remaining

AMS Subject Classifications. Primary 5550; Secondary 5534.

Key Words and Phrases. Postnikov system, stable $k$-invariant, Steenrod algebra, Hopf algebra, Massey-Peterson fundamental sequence. 
maps and modules are as in $\left[5\right.$, p. 63]. In particular $Y^{\prime \prime}=\operatorname{ker} \Omega f^{*}, Z^{\prime}$ and $\Omega Z^{\prime}$ are coker $f^{*}$ and coker $\Omega f^{*}$ respectively.

Using (1) and (2) we associate with each homogeneous element $x \in X^{\prime}(\Omega \xi)$ an element $w \in \Omega Z^{\prime}$ as follows. Let $y \in \Omega Y$ such that $\alpha^{\pi}(y)=x$. Let $t \in Y$ such that $\sigma_{B_{0}}(t)=y$. Since $\sigma_{B} f^{*}(t)=0, f^{*}(t)=\lambda z$ for some $z \in Z$. Let $w=\sigma^{\prime} \rho(z)$. Note that the calculation of $w$ just involves the Adem relations.

Proposition 1. w is a unique element of $\Omega Z^{\prime}$.

Proof. $\sigma_{B_{0}}$ is a map of degree -1 and $\lambda$ doubles degrees. Hence $\rho f^{*} \mid \sigma_{B_{0}}^{-1}\left(\lambda Y^{\prime \prime}\right)=0$. This and looking at the choices involved in the definition of $w$ give the result.

THEOREM A. There exists an element $e \in P(\Omega \xi)$ such that $\Omega i^{*}(e)=x$ and $\bar{\mu}_{2}(e)=q(w \otimes w)$. Here $q$ is the map

$$
\Omega p^{*} \otimes \Omega p^{*}: R(\Omega \xi \times \Omega \xi) \rightarrow P(\Omega \xi \times \Omega \xi) .
$$

The notation is $[5$, p. 63]. The proof uses the Serre spectral sequence in a manner similar to but more involved than arguments of [2] and [7].

REMARKS. 1. Theorem A amounts to calculating the homomorphism

$$
X^{\prime}(\Omega \xi) / \mathrm{im} \sigma_{3} \rightarrow R(\Omega \xi \times \Omega \xi) / \mathrm{im} \bar{\mu}_{1}
$$

in the exact sequence at the bottom of p. 63 [5]. ( $\mu_{i}$ should be replaced by $\bar{\mu}_{i}, i=1,2$, there.)

2. If degree $x$ is odd, then $w=0$. If degree $x$ is even, it is quite possible for $w=0$ and not have $x \in \operatorname{im} \sigma_{3}$. An example is given by $B=K\left(Z_{2}, 2\right), F=K\left(Z_{2}, 7\right)$ and $k$-invariant $\mathrm{Sq}^{4} \mathrm{Sq}^{2}$. This example was also discovered by Massey.

3. Let $\left\{x_{i}\right\}$ be a homogeneous $Z_{2}$-basis for $X^{\prime}(\Omega \xi)$. Let $\left\{e_{i}\right\}$ $\subset P(\Omega \xi)$ satisfy Theorem A with $\Omega i^{*}\left(e_{i}\right)=x_{i}$. Then, by results of [4] and [5], $\{1\} \cup\left\{e_{i}\right\}$ form a simple system of generators for $H^{*}(\Omega E)$ as an algebra over $R$. Thus Theorem A calculates the coproduct of $H^{*}(\Omega E)$ considered as coalgebra over $R$. ( $R$ acts on $H^{*}(\Omega E) \otimes H^{*}(\Omega E)$ via $q \mu_{1}$.)

4. Let $\left\{x_{i}\right\}$ and $\left\{e_{i}\right\}$ be as in Remark 3. Let $\theta \in A$ and consider $\sum x_{j}=\Omega i^{*}\left(\theta e_{i}\right)$. Then $\left(\theta e_{i}+\sum e_{j}\right)=\Omega p^{*}(r)$ for a unique $r \in R$. The naturality of fundamental sequences with respect to loop multiplication and suspension gives much information about $r$. For example a unique element $[r] \in R / S$ is determined by the formula

$$
q \bar{\mu}_{1}([r])=\bar{\mu}_{2}\left(\theta e_{i}+\sum e_{j}\right) .
$$


Here $S \subset R$ is the $A$-submodule of primitives and $\bar{\mu}_{1}: R / S \rightarrow R \otimes R$ is considered as an $A$-map. It is well known to be a monomorphism. A similar formula can be obtained using suspension. We remark that if $F$ and $B$ are 2-connected and $R$ is an exterior algebra over $Z_{2}$, then such formulae and the knowledge of $P\left(\Omega^{2} \xi\right)$ as an $A$-module permit a complete calculation of $P(\Omega \xi)$ as an $A$-module. We defer details to a longer paper.

\section{REFERENCES}

1. J. F. Adams, On the non-existence of elements of Hopf invariant one, Ann. of Math. (2) 72 (1960), 20-104. MR 25 \#4530.

2. J. R. Harper, Stable secondary cohomology operations, Comment. Math. Helv. 44 (1969), 341-353.

3. L. Kristensen, On the cohomology of two-stage Postnikov systems, Acta Math. 107 (1962), 73-123. MR 25 \#4525.

4. W. S. Massey and F. P. Peterson, The cohomology structure of certain fibre spaces. I, Topology 4 (1965), 47-65. MR 32 \#6459.

5. - The mod 2 cohomology structure of certain fibre spaces, Mem. Amer. Math. Soc. No. 74 (1967). MR 37 \#2226.

6. R. J. Milgram, The cohomology over the Steenrod algebra of some two stage Postnikov systems, Quart. J. Math. 20 (1969), 161-169.

7. F. P. Peterson, A note on H-spaces, Bol. Soc. Mat. Mexicana (2) 4 (1959), 3031. MR 22 \#11393.

8. L. Smith, The cohomology of stable two stage Postnikov systems, Illinois J. Math. 11 (1967), 310-329. MR 34 \#8406.

The University of Rochester, Rochester, New York 14627 